\section{Concentración de fluoruro en aguas embotelladas comercializadas en Chile: importancia en caries y fluorosis dental}

\author{
CONSTANZA E. FERNÁNDEZ ${ }^{1, a}$, \\ RODRIGO A. GIACAMAN ${ }^{2, b}$, JAIME A. CURY ${ }^{1, b}$
}

\section{Fluoride concentration in bottled waters sold in Chile}

\begin{abstract}
Background: Bottled water consumption has currently increased and their fluoride $(F)$ concentration may not be ideal in terms of caries benefit or risk of fluorosis. While low concentrations would have little anticaries effect, high $F$ concentration would increase the risk of dental fluorosis. Aim: To measure F concentration in bottled waters sold in Chile. Material and Methods: Thirty bottles of water were purchased (15 sold as mineral water, six sold as purified water and nine as flavored water). Samples were analyzed in duplicate with a previously calibrated ion-specific electrode. Mean F concentration of each product was calculated and expressed as ppm F (mg F/L). Results: A mean ( $\pm S D)$ concentration of $0.39 \pm 0.42,0.02 \pm 0.006$ and $0.11 \pm 0.18 \mathrm{ppm} F$ for mineral, purified and flavored waters respectively, was found. Three samples were within the optimal F concentration recommended for drinking water in Chile, which ranges from 0.6 to $1.0 \mathrm{ppm} F$. Two were above such concentration and the others below. Only two waters displayed F concentration information in the label, which was corroborated by the analysis. Conclusions: Only 10\% of the bottled waters commercialized in Chile have potential to prevent caries. The F concentration in most of them does not represent an increased risk of fluorosis.
\end{abstract}

(Rev Med Chile 2014; 142: 623-629)

Key words: Dental caries; Drinking water; Fluorosis, dental; Mineral waters.
'Facultad de Odontología de Piracicaba. Universidad Estatal de Campinas, São Paulo, Brasil. 2Unidad de Cariología. Departamento de Rehabilitación Buco-máxilofacial, Universidad de Talca, Talca, Chile.

${ }^{a}$ Cirujano Dentista. DDS. PhD student. Becaria Becas Chile.

${ }^{b}$ Cirujano Dentista. DDS. PhD.

Este estudio fue parcialmente financiado por FUNCAMP (Conv. 65/95 and 4252) a JAC sin tener ninguna influencia en la investigación. CEF es Becaria Becas Chile (CONICYT), Los autores declaran no tener conflicto de intereses.

Recibido el 21 de diciembre 2013, aceptado el 15 de abril de 2014.

Correspondencia a: Constanza E. Fernández G. Faculdade de Odontologia de Piracicaba

CP 52 - 13414-903 Piracicaba SP, Brasil.

Teléfono: 55-19-21065303

cfernandez.go@gmail.com
E 1 agua es la fuente más común y de mayor acceso a fluoruros $(\mathrm{F})^{1,2}$, convirtiéndose en una importante estrategia poblacional con eficacia probada para el control de caries ${ }^{3}$. El agua fluorada es un vehículo eficiente para la entrega de $\mathrm{F}$ en baja concentración y en alta frecuencia ${ }^{4}$, lo que permite mantener bajas concentraciones constantes, garantizando así efectividad a todas las edades para el control de caries dental. Además considerando que tanto los beneficios como los riesgos de la ingestión de $\mathrm{F}$ están relacionados con la dosis administrada, la concentración de $\mathrm{F}$ es un importante parámetro de calidad del agua de beber. Así, la mantención de niveles seguros de $\mathrm{F}$ en el agua de beber es una medida esencial para el logro de los objetivos de esta medida de salud pública ${ }^{5}$.

Si bien el consumo de agua fluorada en concentraciones óptimas reduce la prevalencia de caries dental, su consumo durante el período de desarrollo dentario puede aumentar la prevalencia de fluorosis dental en grados leves y muy leves ${ }^{2,3,6}$. Dado que la ocurrencia de fluorosis es dosisdependiente $\mathrm{e}^{7,8}$ y que la cantidad de $\mathrm{F}$ varía pues se pueden obtener $\mathrm{F}$ desde diversas fuentes, se ha intentado determinar una concentración "óptima" que permita obtener el máximo beneficio anticaries con un mínimo riesgo de fluorosis dentaria.

En Chile, la concentración óptima recomendada en agua de beber natural o artificialmente 
Tabla 1. Tipos de aguas analizadas, origen y concentración de fluoruro encontrada

\begin{tabular}{|c|c|c|c|c|}
\hline Tipos de agua & $\begin{array}{l}\text { Fluoruro enc } \\
\text { Media } \pm \text { DE }\end{array}$ & $\begin{array}{l}\text { trado (mg F/L) } \\
\text { Valor min - máx }\end{array}$ & $\mathbf{n}$ & Origen \\
\hline Mineral & $0,39 \pm 0,42$ & $<0,02-1,24$ & 15 & Chile, Alemania, Argentina, Francia, Italia \\
\hline Purificada & $0,02 \pm 0,006$ & $<0,01-0,03$ & 6 & Chile \\
\hline Saborizada & $0,11 \pm 0,18$ & $<0,02-0,59$ & 9 & Chile \\
\hline
\end{tabular}

fluorada es entre 0,6 y $1,0 \mathrm{ppm} \mathrm{F}^{9}$ y se estima que la cobertura nacional de agua potable fluorada sería de $82,3 \%{ }^{10}$.La fluoración del agua comenzó en Valparaíso en $1986^{11}$ e implementado progresivamente en 14 de 15 regiones, excluyendo la Región del Bío-Bío, en donde no se ha adoptado esta medida. La percepción sobre la calidad y seguridad del agua potable tendría importantes implicancias en la exposición adecuada a $\mathrm{F}^{1}$. Así, la desconfianza de algunas personas en relación a que beber agua potable pudiese tener repercusiones negativas en su salud que ha llevado a que cada vez mayor número de personas prefieran beber agua embotellada ${ }^{12,13}$. En Chile y el mundo, el consumo de agua embotellada se ha incrementado de forma importante en los últimos años ${ }^{12,14}$ reemplazando o suplementando el consumo de agua potable. Este aumento posiciona al agua embotellada como una importante fuente de consumo de $\mathrm{F}$ en algunas poblaciones en las que este tipo de fuente de agua contiene el ion. De esta manera, su concentración podría tener importantes repercusiones en la prevención de caries en todos los grupos etarios, pero también con riesgo de fluorosis en población infantil. Previamente, un estudio en Chile ${ }^{15} \mathrm{y}$ varios internacionales ${ }^{16,17}$ han mostrado concentraciones variables de $\mathrm{F}$, indicando la necesidad de control sobre estos productos ${ }^{5}$.

En Chile no existe una normativa que exija especificar la concentración de F en el envase, por lo tanto, el objetivo del presente estudio fue analizar la concentración de $\mathrm{F}$ en aguas embotelladas comercializadas en Chile y discutir el probable efecto anticaries que ellas tendríanjunto con su potencial riesgo de inducir fluorosis dental.

\section{Material y Método}

\section{Aguas embotelladas}

Entre los meses de junio y julio de 2011, 30 variedades de aguas embotelladas: 15 minerales, 6 purificadas (mineralizadas) y 9 saborizadas fueron adquiridas desde los principales supermercados de cadena nacional en las ciudades de Santiago y Talca (Tabla 1). De estas aguas, 13 eran gasificadas (Tabla 2). Las aguas fueron clasificadas en tres tipos, según la clasificación del Reglamento de Aguas Minerales de Chile ${ }^{18}$ : minerales (aguas de origen natural pudiendo o no ser gasificadas); purificadas (aguas que han recibido algún tipo de tratamiento alterando su composición natural) y saborizadas (aguas en las que ha sido adicionado algún saborizante) (Tabla 1). Fueron seleccionadas todas aquellas variedades encontradas en los estantes de aguas embotelladas, excluyendo las bebidas deportivas. Cada variedad fue adquirida en triplicado con número de lote diferente. Las muestras fueron codificadas por un tercero y los análisis fueron realizados de manera ciega.

\section{Determinación de la concentración de F}

Todas las muestras ( $\mathrm{n}=3$ cada tipo de agua, diferente lote) fueron analizadas en duplicado y manteniendo un código para el análisis (Tabla 2). Todas las muestras fueron tamponadas con TISAB II $(1: 1, v / v)$, agitadas y analizadas con electrodo ion-específico (Orion model 96-09, Orion Research, Cambridge, MA) acoplado a un analizador de iones (Orion EA-940). El electrodo fue previamente calibrado con estándares de 0,031 a $1,0 \mu \mathrm{g}$ $\mathrm{F} / \mathrm{mL}$, también tamponados con TISAB II $(1: 1, \mathrm{v} / \mathrm{v})$ y leídos en triplicado. Una regresión lineal entre concentración de fluoruro $\mathrm{F}$ y $\mathrm{mV}\left(\mathrm{r}^{2}=0,9999\right.$, slope $=55,0)$ permitió determinar la concentración de $\mathrm{F}$ en las muestras. El promedio $(\mathrm{n}=3)$ de la concentración de $\mathrm{F}$ ( \pm desviación estándar, $\mathrm{DE}$ ) de cada variedad, clasificadas por tipo de agua (mineral, purificada y saborizada) fue calculada y expresada como ppm F ( $\mu \mathrm{g} \mathrm{F} / \mathrm{mL}=\mathrm{mg} \mathrm{F} / \mathrm{L})$.

\section{Análisis de los datos}

Se promediaron los valores de cada medición duplicada y luego los obtenidos para cada marca 
Tabla 2. Aguas analizadas y concentración de fluoruro declarada y encontrada (mg F/L)

\begin{tabular}{|c|c|c|c|}
\hline Tipo & $\begin{array}{l}\text { Código } \\
\text { para } \\
\text { análisis }\end{array}$ & $\begin{array}{c}\text { F } \\
\text { declarado } \\
\text { (mg/L) }\end{array}$ & $\begin{array}{c}\text { Fluoruro } \\
\text { encontrado } \\
\text { (mg F/L). } \\
\text { Media } \pm \text { DE } \\
(n=3)\end{array}$ \\
\hline \multirow[t]{15}{*}{ Agua mineral } & 2 & ND & $0,14 \pm 0,026$ \\
\hline & 4 & ND & $0,11 \pm 0,006$ \\
\hline & 5 & 0,3 & $0,33 \pm 0,012$ \\
\hline & 8 & ND & $0,05 \pm 0,006$ \\
\hline & 9 & ND & $0,02 \pm 0,006$ \\
\hline & 10 & ND & $0,21 \pm 0,012$ \\
\hline & 13 & ND & $0,09 \pm 0,015$ \\
\hline & 16 & ND & $0,36 \pm 0,010$ \\
\hline & $17^{\mathrm{G}}$ & ND & $1,24 \pm 0,051$ \\
\hline & $18^{\mathrm{G}}$ & ND & $0,13 \pm 0,006$ \\
\hline & $19^{\mathrm{G}}$ & 1,2 & $1,28 \pm 0,000$ \\
\hline & 23 & ND & $0,33 \pm 0,006$ \\
\hline & $35^{G}$ & ND & $0,55 \pm 0,007$ \\
\hline & 36 & ND & $1,03 \pm 0,012$ \\
\hline & 40 & ND & $0,05 \pm 0,017$ \\
\hline \multirow[t]{6}{*}{ Agua purificada } & 1 & ND & $0,01 \pm 0,006$ \\
\hline & 7 & ND & $0,02 \pm 0,006$ \\
\hline & 15 & ND & $0,03 \pm 0,006$ \\
\hline & 22 & ND & $0,02 \pm 0,006$ \\
\hline & $38^{G}$ & ND & $0,02 \pm 0,006$ \\
\hline & 39 & ND & $0,02 \pm 0,006$ \\
\hline \multirow[t]{9}{*}{ Agua saborizada } & $3^{G}$ & ND & $0,07 \pm 0,006$ \\
\hline & 6 & ND & $0,04 \pm 0,006$ \\
\hline & $11^{\mathrm{G}}$ & ND & $0,02 \pm 0,006$ \\
\hline & $12^{\mathrm{G}}$ & ND & $0,1 \pm 0,000$ \\
\hline & $14^{\mathrm{G}}$ & ND & $0,59 \pm 0,050$ \\
\hline & $20^{G}$ & ND & $0,07 \pm 0,006$ \\
\hline & $21^{\mathrm{G}}$ & ND & $0,04 \pm 0,006$ \\
\hline & $34^{G}$ & ND & $0,08 \pm 0,006$ \\
\hline & $37^{\mathrm{G}}$ & ND & $0,02 \pm 0,000$ \\
\hline
\end{tabular}

ND: no declarado en el envase. G: producto comercial gasificado. $(\mathrm{n}=3)$ más su DE. Los valores se expresaron descriptivamente en cuanto a su concentración de F y se establecieron comparaciones descriptivas entre productos y por tipo de agua. Los cálculos fueron realizados utilizando el software Excel 2007 para Windows ${ }^{\circledR}$.

\section{Resultados}

La mayoría de las aguas embotelladas analizadas y disponibles en el mercado (50\%) correspondieron a aguas minerales, las restantes se dividieron entre saborizadas $(30 \%)$ y purificadas (20\%). La concentración de $\mathrm{F}$ de las aguas varió entre $<0,02$ y $1,24 \mathrm{ppm} F$, con una media $( \pm \mathrm{DE})$ de $0,24 \pm 0,36 \mathrm{ppm}$ F. La variación promedio entre cada duplicado fue de $1,5 \%$, mostrando reproductibilidad de los resultados. La concentración promedio de las aguas minerales fue $0,39 \pm 0,42$ ppm de F, purificadas $0,02 \pm 0,006$ y saborizadas $0,11 \pm 0,18$ (Tabla 1). Las aguas purificadas presentaron la menor concentración de $F$, siendo este ion casi inexistente en las muestras $(\leq 0,03 \mathrm{ppm}$ F, bajo el límite de sensibilidad del electrodo $)^{19}$.

Dado que la concentración recomendada en el agua de beber en Chile es de 0,6 a 1,0 ppm F, sólo tres muestras (10\%) estaban dentro de este rango (agua $\mathrm{N}^{\circ} 14,35$ y 36$)$, dos $(6,7 \%)$ sobre el límite superior $\left(\mathrm{N}^{\circ} 17\right.$ y 19$)$ y las restantes $(83,3 \%)$ bajo el mínimo (Tabla 2 y Figura 1 ). Se podría considerar que 17 aguas embotelladas (56,7\%) no contienen F, pues mostraron menos de $0,1 \mathrm{ppm}$ de F. Sólo dos productos mostraron información de la concentración de F presente ( $\mathrm{N}^{\circ} 5$ y 19$)$, la que coincidió con la declarada por el fabricante. 93,3\% restante no indicó la presencia de $\mathrm{F}$ en su composición (Tabla 2).

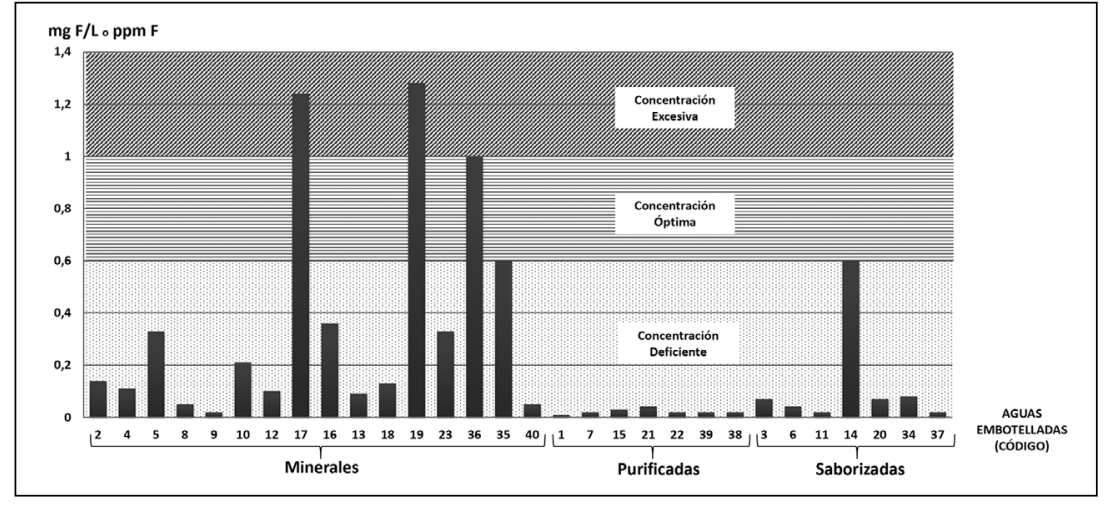

Figura 1. Concentración de fluoruro encontrada y relación con la concentración óptima. En la franja central está representada la concentración óptima de $F$ (entre 0,6 y 1,0 ppm F). En franja inferior niveles bajo el nivel recomendado y en franja superior niveles elevados. 


\section{Discusión}

Una adecuada exposición al F permite aprovechar los beneficios anticaries en pacientes de cualquier edad y con un mínimo riesgo de fluorosis en la población infantil. Actualmente, es sabido que para el control de caries el F presente en el agua de beber actúa local y no sistémicamente. Por un lado, el contacto directo del agua fluorada o alimentos cocidos con ella en la cavidad bucal aumenta las concentraciones de $\mathrm{F}$ en el biofilm oral ${ }^{19,20}$ y en la saliva $^{21}$, generando así una mayor cantidad de $\mathrm{F}$ libre para interferir en el proceso de formación de caries ${ }^{22}$. Adicionalmente, el $\mathrm{F}$ ingerido es excretado en bajas dosis por las glándulas salivales, lo que extiende la acción local del $\mathrm{F}$ por períodos de tiempo mayores en el ecosistema bucal ${ }^{23,24}$. En relación a las aguas embotelladas vendidas en Chile y analizadas en este estudio, sólo $10 \%$ contiene concentración con potencial para prevenir caries si se considera el rango de 0,6 a 1 ppm F como la concentración óptima9 .

Por otra parte, los resultados de este estudio muestran que la gran mayoría de las aguas embotelladas disponibles en el mercado chileno no aumentan el riesgo de fluorosis, ya que $90 \%$ contenía concentraciones de $\mathrm{F}$ menores a $1 \mathrm{ppm}$. Pese a que existe una relación dosis respuesta ${ }^{7,8}$, en general la fluorosis es consecuencia de la ingesta excesiva de $\mathrm{F}$ durante el período de desarrollo dentario $^{25}$. El período crítico de exposición excesiva a $\mathrm{F}$ es desde el nacimiento hasta los 8 años de edad ${ }^{6}$. Respecto a la prevalencia de fluorosis a nivel nacional, el levantamiento epidemiológico del 2007 reveló que 31,2\% de adolescentes de 12 años presentan algún tipo de fluorosis, principalmente grados leves y muy leves ${ }^{26}$. La proporción de fluorosis con compromiso estético en los niños $y$ adolescentes disminuye a medida que la concentración de fluoruro en el agua se acerca al rango de 0,5-0,7 ppm de F, donde la tendencia es a una proporción de fluorosis sin compromiso estético, considerado nivel seguro de flúor, ya sea en agua natural o artificialmente fluorada ${ }^{5}$. No obstante existe un nivel detectable de fluorosis cuando se administran F, los grados leve o muy leve que se asocian a este tipo de exposición a $\mathrm{F}$ tienen un pequeño impacto en la calidad de vida de niños $y$ adolescentes ${ }^{27}$ e incluso, se ha reportado una asociación de fluorosis con un mejoramiento en la calidad de vida ${ }^{28}$. Lo anterior, probablemente se deba a que el aumento en la tasa de fluorosis se asocia a mayor exposición a $\mathrm{F}$ y por ende a un aumento en los efectos benéficos del ion; menor incidencia de caries y con ello menos cuadros de dolor, menor necesidad de terapias complejas y menor demanda de servicios odontológicos.

Según las reglamentaciones internacionales ${ }^{29}$, cuando las aguas embotelladas poseen entre 0,8 a 1 ppm de F deberían indicar en el envase "contiene fluoruro". Sin embargo, de las 3 marcas que contienen esa o mayor concentración, sólo una la declara en el envase (Tabla 2). A diferencia del contenido de sodio que en Chile se encuentra normado por Decreto Supremo No 977/96 de 1999 MINSAL, la normativa chilena no exige que la concentración de F sea presentada en la etiqueta de los productos, más sí que la concentración de F sea inferior a 2 $\mathrm{ppm} \mathrm{F}^{30}$. De ser declarada la concentración F, compradores informados podrían elegir beneficiarse con el F u optar por agua sin contenido de F, lo que podría ser de relevancia en los niños.

Numerosas investigaciones han reportado la concentración de F presente en aguas embotelladas, mostrando preocupación por evaluar y conocer esa información. El método de análisis utilizado en todas ellas es el electrodo ion-selectivo ${ }^{16,31} \mathrm{el}$ cual es considerando de fácil acceso y que presenta un aceptable límite inferior de detección ${ }^{32}$.

A pesar del bajo contenido de $\mathrm{F}$ en las aguas disponibles en el comercio en Chile, al análisis de diferentes lotes del producto, no se encontraron grandes variaciones en la concentración de F, lo que sugiere que el proceso de producción es bien controlado. Los resultados de esta investigación se aproximan a las concentraciones previamente reportadas en aguas minerales de origen chileno ${ }^{15}$. Sin embargo, ninguna de las aguas analizadas en este estudio alcanzó 2 ppm F. Las más altas concentraciones reportadas en dicho estudio se reportaron para las aguas Mamiña (6,61 ppm F) y Chusmiza (7,94 ppm F), las cuales no fueron encontradas en el mercado en el año de este estudio.

Si bien en Chile no se ha descrito el consumo real de agua embotellada en niños, dado el explosivo aumento de la oferta de estos productos en el mercado, es posible elucubrar que al igual que en otros países, el consumo ha aumentado de manera relevante. Información de estudios internacionales indica un aumento del consumo desde el nacimiento, independiente de nivel de ingresos ${ }^{33}$ raza $^{12}$. Debido a que en muchos 
casos, las aguas embotelladas no contienen o contienen cantidades insuficientes de F, como se muestra en este trabajo, se ha planteado que los médicos deberían proporcionar orientación a las familias con respecto a la seguridad, bajo costo y los beneficios de salud dental de beber agua potable fluorada ${ }^{34}$, desalentando así el consumo de agua embotellada. Es importante señalar, que pese a que las dosis de $\mathrm{F}$ podrían variar considerablemente al consumir agua embotellada o agua potable fluorada, la población chilena se encuentra expuesta a otras fuentes de $\mathrm{F}$ en dosis altas, como son los dentífricos. A ese respecto, los productos comerciales para adultos y para niños ${ }^{35,36}$ cuentan con la cantidad de F activo (soluble) señalada por el fabricante. Se requiere más investigación para determinar si el reemplazo del consumo de agua potable fluorada por el agua embotellada sin F, pero manteniendo la exposición a las dosis contenidas en los dentífricos tiene un impacto real en la ocurrencia de caries de la población. Es importante destacar que la población que vive en una región con agua fluorada, aun cuando no consuma agua potable para beber, continúa beneficiándose por la incorporación de $\mathrm{F}$ cuando los alimentos son preparados con dicha agua ${ }^{37}$.

Dada la gran disparidad en el contenido de $\mathrm{F}$ en las aguas disponibles en Chile, se sugiere que la concentración de F debería ser declarada en el envase de los fabricantes. En el marco de la coyuntura actual por la legislación sobre el rotulado de la composición de alimentos que vive el país, este tema podría incluirse en las nuevas normativas en preparación. Si se dispone de información clara sobre las cantidades de F y su significancia tanto en cuanto a riesgos como a beneficios de su uso, las personas podrán escoger los productos no sólo por sus características organolépticas, sino por sus efectos en la salud oral que ellos traen aparejados. De especial interés, el uso de aguas embotelladas en niños pequeños puede tener un doble efecto negativo; por una parte el escoger aguas sin $\mathrm{F}$ en su composición priva del beneficio anticaries del $\mathrm{F}$ y por otra el consumo de aguas embotelladas con cantidades excesivas del ion aumenta el riesgo de fluorosis. Si para un niño de 1 año (10 kilos) se prepara leche en polvo usando agua fluorada de 1,24 ppm F (3 mamaderas $/ 200 \mathrm{~mL}$ ) la ingesta de F sería $0,074 \mathrm{mg} \mathrm{F} / \mathrm{kg} /$ día, valor superior de lo considerado en riesgo de fluorosis ${ }^{38}$. En Estados Unidos de Norteamérica 50\% de las madres dice usar agua embotellada para la preparación de las formulas infantiles ${ }^{39}$.

En conclusión, las aguas embotelladas disponibles en Chile contienen en general bajas concentraciones de F, independientemente de su tipo y su consumo no garantiza beneficios comparables con beber agua potable fluorada. Algunas marcas incorporan F, sin embargo, las concentraciones son altamente variables y la legislación del país no obliga ni al control de las dosis ni al rotulado de los contenidos.

Agradecimientos: Los autores agradecen el soporte técnico del Sr. Waldomiro Vieira Filho, del Laboratorio de Bioquímica Oral de la FOPUNICAMP, Brasil.

\section{Referencias}

1. Scherzer T, Barker JC, Pollick H, Weintraub JA. Water consumption beliefs and practices in a rural Latino community: implications for fluoridation. J Public Health Dent 2010; 70 (4): 337-43.

2. Ellwood R, Ferjeskov O, Cury JA, Clarkson B. Fluorides in Caries Control. In: Kidd Fa, editor. Dental Caries, The disease and its clinical management: Oxford 2008; p. 288322.

3. McDonagh MS, Whiting PF, Wilson PM, Sutton AJ, Chestnutt I, Cooper J, et al. Systematic review of water fluoridation. BMJ 2000; 321 (7265): 855-9.

4. Kumar JV. Is water fluoridation still necessary? Adv Dent Res 2008; 20 (1): 8-12.

5. Frazao P, Peres MA, Cury JA. Drinking water quality and fluoride concentration. Rev Saude Publica 2011; 45 (5): 964-73.

6. Mascarenhas AK. Risk factors for dental fluorosis: a review of the recent literature. Pediatr Dent 2000; 22 (4): 269-77.

7. Dean HT. Fluorine and dental caries. Am J Orthod 1947; 33 (2): 49-67.

8. Cury J. Uso do flúor e controle da Cárie como Doença. En: Baratieri, editor. Odontologia restauradora-Fundamentos e Possibilidades; São Paulo, Brasil.Ed. Santos; 2001. p. 31-68.

9. MINSAL. Normas de Uso de Fluoruros en la Prevención Odontológica. Norma General Técnica No 105 In: Departamento de Salud Bucal. División de prevención y control de Enfermedades SdSP, editor: Gobierno de Chile, Ministerio de Salud de Chile 2008; p. 15-20.

10. Gómez S, Fernández O. Fluoración del Agua Potable, 
Experiencia en Chile. En: Gómez Soler G, editor. Fluorterapia en Odontologia Fundamentos y Aplicaciones Clínicas 2010; p. 162-77.

11. Badenier O, Cueto A, Mariño R, Acevedo R. Situación de Salud Bucodental y algunos Factores de riesgo en Adultos de 35 a 44 años de la Región de Valparaíso. Revista de la Facultad de Odontología Universidad de Valparaíso 2009; 1103-8.

12. Huerta-Sáenz L, Irigoyen $M$, Benavides J, Mendoza M. Tap or Bottled Water: Drinking Preferences Among Urban Minority Children and Adolescents. J Community Health 2012; 37 (1): 54-8.

13. Doria MF. Bottled water versus tap water: understanding consumers' preferences. J Water Health 2006; 4 (2): 2716.

14. ANBER. Associación nacional de Bebidas Refrescantes. Comunicado de Prensa. 2011.

15. Barrios C, Urzúa I, Mondaca G, Yevenes I. Cuantificación del ion fluor de nueve aguas minerales en Chile. Revista Dental de Chile 2006; 97 (1): 3-6.

16. Villena RS, Borges DG, Cury JA. [Evaluation of fluoride content of bottled drinking waters in Brazil]. Rev Saude Publica 1996; 30 (6): 512-8.

17. Johnson SA, De Biase C. Concentration levels of fluoride in bottled drinking water. J Dent Hyg 2003; 77 (3): 1617.

18. Mercurio EEE. Mercado nacional: Tomar agua mineral está de moda. El Mercurio [Internet]. 2005. Available from: http://www.edicionesespeciales.elmercurio.com/ destacadas/detalle/index.asp?idnoticia $=012312200502$ $1 \mathrm{X} 0060011$ \&idcuerpo=482. [Consultado el $18 \mathrm{de} \mathrm{sep-}$ tiembre de 2011].

19. Whitford GM. The metabolism and toxicity of fluoride. Monogr Oral Sci 1996; 16 Rev 2: 1-153.

20. Nobre dos Santos M, Cury JA. Dental plaque fluoride is lower after discontinuation of water fluoridation. Caries Res 1988; 22 (5): 316-7.

21. Oliveby A, Twetman S, Ekstrand J. Diurnal fluoride concentration in whole saliva in children living in a high- and a low-fluoride area. Caries Res 1990; 24 (1): 44-7.

22. Cury JA, Tenuta LMA. Uso racional de fluoreto. In: Feldens CA; Kramer PF. (Org.). Cárie dentária na infância: uma abordagem contemporânea. 1ed.São Paulo: Santos, 2013, v. , p. 147-58.

23. Tenuta LM, Cury JA. Fluoride: its role in dentistry. Braz Oral Res 2010; 24 Suppl 1: 9-17.

24. Cury JA, Tenuta LM. How to maintain a cariostatic fluoride concentration in the oral environment. Adv Dent Res 2008; 20 (1): 13-6.

25. Denbesten P, Li W. Chronic fluoride toxicity: dental fluorosis. Monogr Oral Sci 2011; 22: 81-96.

26. Soto L, Tapia R, col. Diagnóstico Nacional de Salud Bucal del Adolescente de 12 años y Evaluación del Grado de Cumplimiento de los Objetivos Sanitarios de Salud Bucal 2000-2010. Chile 2007.

27. Chankanka O, Levy SM, Warren JJ, Chalmers JM. A literature review of aesthetic perceptions of dental fluorosis and relationships with psychosocial aspects/oral healthrelated quality of life. Community Dent Oral Epidemiol 2010; 38 (2): 97-109.

28. Peres KG, Peres MA, Araujo CL, Menezes AM, Hallal PC. Social and dental status along the life course and oral health impacts in adolescents: a population-based birth cohort. Health Qual Life Outcomes 2009; 7: 95.

29. FAO-CODEX-STAN108-1981. Comisión del codex alimentarius. FAO. OMS sobre Normas Alimentarias. Norma General para Aguas Envasadasl/Embotelladas distintas de Aguas Minerales Naturales. http:// www.fao.org/docreplmeeting/005/X0818S/x0818s0b. Htm\#TopOfPage,1998.; Rev. 1-1997. [Consultado el 18 de septiembre de 2011].

30. Ministerio de Salúd GdC. Reglamento de aguas Minerales Decreto. No 106 de 1997 actualizado 2001. Available from: http://www.redsalud.gov.cl/archivos/alimentosynutricion/inocuidad/aguasminerales1.pdf. [Consultado el 8 de agosto de 2011].

31. Quock RL, Chan JT. Fluoride content of bottled water and its implications for the general dentist. Gen Dent 2009; 57 (1): 29-33.

32. Martínez-Mier EA, Cury JA, Heilman JR, Katz BP, Levy SM, Li Y, et al. Development of gold standard ionselective electrode-based methods for fluoride analysis. Caries Res 2011; 45 (1): 3-12.

33. Sriraman NK, Patrick PA, Hutton K, Edwards KS. Children's drinking water: parental preferences and implications for fluoride exposure. Pediatr Dent 2009; 31 (4): 310-5.

34. Hobson WL, Knochel ML, Byington CL, Young PC, Hoff CJ, Buchi KF. Bottled, filtered, and tap water use in Latino and non-Latino children. Arch Pediatr Adolesc Med 2007; 161 (5): 457-61.

35. Giacaman RA, Carrera CA, Muñoz-Sandoval C, Fernández $\mathrm{C}$, Cury JA. Fluoride content in toothpastes commercialized for children in Chile and discussion on professional recommendations of use. Int J Paediatr Dent 2013; 23 (2): 77-83.

36. Carrera CA, Giacaman RA, Muñoz-Sandoval C, Cury JA. Total and soluble fluoride content in commercial dentifrices in Chile. Acta Odontol Scand 2012; 70 (6): 583-8.

37. Casarin RC, Fernandes DR, Lima-Arsati YB, Cury JA. 
Fluoruro en aguas embotelladas y su importancia en caries y fluorosis - C. E. Fernández et al

[Fluoride concentrations in typical Brazilian foods and in infant foods]. Rev Saude Publica 2007; 41 (4): 549-56.

38. Burt BA. The changing patterns of systemic fluoride intake. J Dent Res 1992; 71 (5): 1228-37.
39. CDC. Centers for Disease Control. Infant feeding Infant practiques study II table 397 [Internet]. 2009. Available from: http://www.cdc.gov/ifps/results/ch3/table3-97. htm. [Consultado el 3 de noviembre de 2011]. 